\title{
CATEGORY OF MAPS AND GOTTLIEB SETS FOR MAPS, AND THEIR DUALS
}

\author{
YEON SOO YOON*
}

\begin{abstract}
In this paper, we introduce and study the concepts of $W C_{k}^{f}$-spaces with respect to spaces which are generalized concepts of $C_{k}^{f}$-spaces for maps, and introduce the dual concepts of $W C_{k}^{f}$ spaces with respect to spaces and obtain some dual results.
\end{abstract}

\section{Introduction}

Throughout this paper, a space means a space of the homotopy type of a locally finite connected $C W$ complex. All maps shall mean continuous functions. It is known that any space $X$ is filtered by the projective spaces of $\Omega X$ by a result of Milnor [8] and Stasheff [10];

$$
\Sigma \Omega X=P^{1}(\Omega X) \hookrightarrow P^{2}(\Omega X) \hookrightarrow \cdots \hookrightarrow P^{\infty}(\Omega X) \simeq X .
$$

For each $k$, let $e_{k}^{X}: P^{k}(\Omega X) \rightarrow P^{\infty}(\Omega X) \simeq X$ be the natural inclusion. Let $f: A \rightarrow X$ be a map. A space $X$ is called [5] a $C_{k}^{f}$-space if the inclusion $e_{k}^{X}: P^{k}(\Omega X) \rightarrow X$ is $f$-cyclic. It is known [5] that a space $X$ is a $C_{k}^{f}$-space for a map $f: A \rightarrow X$ if and only if $G^{f}(Z, X)=[Z, X]$ for any space $Z$ with cat $Z \leq k$. For any spaces $Z, X$, we define mapcat $(Z, X) \leq k$ if for any map $g: Z \rightarrow X$, cat $g \leq k$. It is known that if cat $Z \leq k$, then mapcat $(Z, X) \leq k$, but the converse does not hold(see Example 2.6).

In this paper, we introduce the concepts of $W C_{k}^{f}$-spaces with respect to spaces which are generalizations of $C_{k}^{f}$-spaces for maps [5] and study some properties of $W C_{k}^{f}$-spaces with respect to spaces. We show that for a space $Z$ with mapcat $(Z, X) \leq k$, a space $X$ is a $W C_{k}^{f}$-space with

Received January 10, 2013; Accepted January 21, 2013.

2010 Mathematics Subject Classification: Primary 55P45, 55P35.

Key words and phrases: $f$-cyclic maps, categories of maps, $p$-cocyclic maps, cocategories of maps.

The author was supported by Hannam University Research Fund, 2012. 
respect to $Z$ if and only if $G^{f}(Z, X)=[Z, X]$. Let $f: A \rightarrow X$ and $g: B \rightarrow Y$ be any maps and $Z$ a space with mapcat $(Z, X) \leq k$. Then we show that the product space $X \times Y$ is a $W C_{k}^{f \times g}$-space with respect to $Z$ if and only if $X$ is a $W C_{k}^{f}$-space with respect to $Z$ and $Y$ is a $W C_{k}^{g}$-space with respect to $Z$. We also introduce the dual concepts of $W C_{k}^{f}$-spaces with respect to spaces and obtain some dual results.

\section{2. $W C_{k}^{f}$-spaces with respect to spaces}

The $L S$ category of $X$ [3], denoted cat $X$, is the least integer $k$ such that $X$ is the union of $k+1$ open sets $U_{i}$, each contractible in $X$. We now recall the following Ganea's theorems [3].

Theorem 2.1. ([3],[4]) The category cat $X \leq k$ if and only if $e_{k}^{X}$ : $P^{k}(\Omega X) \rightarrow X$ has a right homotopy inverse.

The definition of LS category extends from spaces to continuous maps as follows. Let $g: X \rightarrow Y$ be a map. The $L S$ category of $g$ [3], denoted cat $g$ is the least integer $k$ such that $X$ is the union of $k+1$ open sets $U_{i}$ for which the restriction of $g$ to each $U_{i}$ is homotopic to a constant map $U_{i} \rightarrow *$. Note that cat $X=$ cat $1_{X}$.

Theorem 2.2. [3] Let $g: Z \rightarrow X$ be a map. Then the category cat $g \leq k$ if and only if there is a map $\bar{g}: Z \rightarrow P^{k}(\Omega X)$ such that $e_{k}^{X} \circ \bar{g} \sim g: Z \rightarrow X$, where $e_{k}^{X}: P^{k}(\Omega X) \rightarrow X$ is the natural inclusion.

Definition 2.3. Let $Z, X$ be any two spaces. The mapcategory of mapping space from $Z$ to $X$ is less than equal to $k$, mapcat $(Z, X) \leq k$, means that for any map $g: Z \rightarrow X$, cat $g \leq k$.

It is clear that mapcat $(Z, X) \leq k$ if and only if $\left(e_{k}^{X}\right)_{\#}:\left[Z, P^{k}(\Omega X)\right] \rightarrow$ $[Z, X]$ is an epimorphism.

The following propositions say that a relationship between category of a space and mapcategory of a mapping space.

Proposition 2.4. cat $Z \leq k$ if and only if for any space $X$, mapcat $(Z$, $X) \leq k$.

Proof. Suppose that cat $Z \leq k$. Then there is a map $s_{k}^{Z}: Z \rightarrow$ $P^{k}(\Omega Z)$ such that $e_{k}^{Z} \circ s_{k}^{Z} \sim 1$. Let $X$ be a space and $g: Z \rightarrow X$ a map. We see $e_{k}^{X} \circ P^{k}(\Omega g) \sim g \circ e_{k}^{Z}$ by the naturality of the construction of 
$P^{k}(\Omega Z)$ as is shown in the following homotopy commutative diagram;

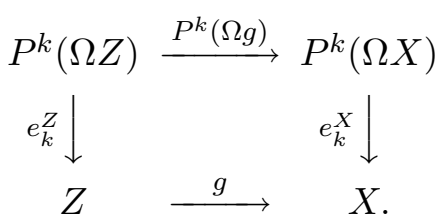

Thus we have a map $\bar{g}=P^{k}(\Omega g) \circ s_{k}^{Z}: Z \rightarrow P^{k}(\Omega X)$ such that $e_{k}^{X} \circ \bar{g} \sim g$. Thus we know mapcat $(Z, Y) \leq k$. On the other hand, suppose that for any space $X$, the mapcategory mapcat $(Z, X) \leq k$. Taking $X=Z$ and $g=1_{Z}$, then we know that cat $Z \leq k$.

Proposition 2.5. cat $X \leq k$ if and only if for any space $Z$, mapcat $(Z$, $X) \leq k$.

Proof. Suppose that cat $X \leq k$. Then there is a map $s_{k}^{X}: X \rightarrow$ $P^{k}(\Omega X)$ such that $e_{k}^{X} \circ s_{k}^{X} \sim 1$. Let $Z$ be a space and $g: Z \rightarrow X$ a map. Then we have $e_{k}^{X} \circ\left(s_{k}^{X} \circ g\right) \sim 1_{X} \circ g \sim g$ and mapcat $(Z, X) \leq k$. On the other hand, suppose that for any space $Z$, the mapcategory mapcat $(Z, X) \leq k$. Taking $Z=X$ and $g=1_{X}$, then we know that cat $X \leq k$.

In general, if cat $Z \leq k$, then mapcat $(Z, X) \leq k$ for a space $X$, but the converse does not hold by the following example.

EXAMPLE 2.6. It is well known fact that cat $\mathbb{C} P^{n}=n$. Thus if we take $X=\mathbb{C} P^{k}$ and $Z=\mathbb{C} P^{k+1}$, then we know, from Proposition 2.5, that mapcat $(Z, X) \leq k$, but cat $Z=k+1$.

Let $f: A \rightarrow X$ be a map. A based map $g: B \rightarrow X$ is called $f$-cyclic [12] if there is a map $\phi: A \times B \rightarrow X$ such that the diagram

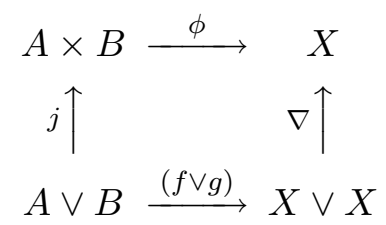

is homotopy commute, where $j: A \vee B \rightarrow A \times B$ is the inclusion and $\nabla: X \vee X \rightarrow X$ is the folding map. Clearly, $g$ is $f$-cyclic iff $f$ is $g$ cyclic. In the case, $f=1_{X}: X \rightarrow X, g: B \rightarrow X$ is called cyclic [15]. We denote the set of all homotopy classes of $f$-cyclic maps from $B$ to $X$ by $G^{f}(B, X) \subset[B, X]$ which is called the Gottlieb set for a map $f: A \rightarrow X$. If $f=1_{X}: X \rightarrow X$, then we recover the Gottlieb set $G(B, X)=G^{1 X}(B, X)$ defined by Varadarajan [11]. In general, 
$G(B, X) \subset G^{f}(B, X) \subset[B, X]$ for any spaces $A, B, X$ and any map $f: A \rightarrow X$.

It is shown [14] that $G\left(S^{5}, S^{5} \times S^{5}\right) \cong 2 \mathbb{Z} \oplus 2 \mathbb{Z} \neq G^{i_{1}}\left(S^{5}, S^{5} \times S^{5}\right) \cong$ $2 \mathbb{Z} \oplus \mathbb{Z} \neq\left[S^{5}, S^{5} \times S^{5}\right] \cong \mathbb{Z} \oplus \mathbb{Z}$. It is introduced [5] that a space $X$ is called a $C_{k}^{f}$-space if the inclusion $e_{k}^{X}: P^{k}(\Omega X) \rightarrow X$ is $f$-cyclic.

Lemma 2.7. Let $f: A \rightarrow X$ be a map. Then $g: B \rightarrow X$ is $f$-cyclic if and only if $(g)_{\#}([Z, B]) \subset G^{f}(Z, X)$ for any space $Z$.

Proof. Suppose that $g: B \rightarrow X$ is $f$-cyclic. Let $Z$ be a space and $\theta: Z \rightarrow B$ a map. Since $g: B \rightarrow X$ is $f$-cyclic, there is a map $G: A \times$ $B \rightarrow X$ such that $G j \sim \nabla(f \vee g)$, where $j: A \vee B \rightarrow A \times B$ is the inclusion and $\nabla: X \vee X \rightarrow X$ is the folding map. Then $\phi=G(1 \times \theta): A \times Z \rightarrow X$ satisfies $\phi j \sim \nabla(f \vee g \theta)$. Thus we have $g_{\#}([Z, B]) \subset G^{f}(Z, X)$ for any space $Z$. On the other hand, taking $Z=B$ and $1_{B}: B \rightarrow B \in[B, B]$. Since $g \sim g_{\#}\left(1_{B}\right) \in G^{f}(B, X), g: B \rightarrow X$ is $f$-cyclic.

Theorem 2.8. [5] $A$ space $X$ is a $C_{k}^{f}$-space for a map $f: A \rightarrow X$ if and only if $G^{f}(Z, X)=[Z, X]$ for any space $Z$ with cat $Z \leq k$.

EXAMPLE 2.9 .

(1) The torus $T^{k}$ is a space with $\operatorname{mapcat}\left(T^{k}, X\right) \leq k$. It is well known fact that cat $T^{k}=k$. Thus we know, from Proposition 2.4, that $\operatorname{mapcat}\left(T^{k}, X\right) \leq k$, that is, $\left(e_{k}^{X}\right)_{\#}:\left[T^{k}, P^{k}(\Omega X)\right] \rightarrow\left[T^{k}, X\right]$ is an epimorphism.

(2) If a space $Z$ satisfy cat $Z \leq k$, then $Z$ is also a space with mapcat( $Z$, $X) \leq k$ from Proposition 2.4.

Definition 2.10. Let $f: A \rightarrow X$ be a map and $Z$ a space with mapcat $(Z, X) \leq k$. Then a space $X$ is called a $W C_{k}^{f}$-space with respect to a space $Z$ if $\left(e_{k}^{X}\right)_{\#}\left(\left[Z, P^{k}(\Omega X]\right) \subset G^{f}(Z, X)\right.$, where $e_{k}^{X}: P^{k}(\Omega X) \rightarrow$ $X$ is the natural inclusion.

Theorem 2.11. Let $f: A \rightarrow X$ be a map and $Z$ space with mapcat $(Z$, $X) \leq k$. A space $X$ is a $W C_{k}^{f}$-space with respect to $Z$ if and only if $G^{f}(Z, X)=[Z, X]$.

Proof. Suppose that $X$ is a $W C_{k}^{f}$-space with respect to $Z$. Since mapcat $(Z, X) \leq k,\left(e_{k}^{X}\right)_{\#}:\left[Z, P^{k}(\Omega X)\right] \rightarrow[Z, X]$ is an epimorphism. Since $X$ is a $W C_{k}^{f}$-space with respect to $Z,[Z, X]=\left(e_{k}^{X}\right)_{\#}\left(\left[Z, P^{k}(\Omega X)\right]\right)$ $\subset G^{f}(Z, X)$ and $G^{f}(Z, X)=[Z, X]$.

Conversely, assume that $G^{f}(Z, X)=[Z, X]$. Thus we know $\left(e_{k}^{X}\right)_{\#}([Z$, $\left.\left.P^{k}(\Omega X)\right]\right)=[Z, X] \subset G^{f}(Z, X)$ and $X$ is a $W C_{k}^{f}$-space with respect to $Z$. 
We have the following corollary from Theorem 2.8 and Example 2.9.

Corollary 2.12. $X$ is a $C_{k}^{f}$-space if and only if for each space $Z$ with cat $Z \leq k, X$ is a $W C_{k}^{f}$-space with respect to $Z$.

Theorem 2.13. Let $f: A \rightarrow X$ and $g: B \rightarrow Y$ be any maps and $Z$ a space with mapcat $(Z, X) \leq k$. Then the product space $X \times Y$ is a $W C_{k}^{f \times g}$-space with respect to $Z$ if and only if $X$ is a $W C_{k}^{f}$-space with respect to $Z$ and $Y$ is a $W C_{k}^{g}$-space with respect to $Z$.

Proof. Suppose $X \times Y$ is a $W C_{k}^{f \times g}$-space with respect to $Z$. It is known [5] that $G^{f \times g}(Z, X \times Y) \cong G^{f}(Z, X) \times G^{g}(Z, Y)$ for any space $Z$. Since $Z$ is a space with mapcat $(Z, X) \leq k$, we have, from Theorem 2.11 , that $G^{f}(Z, X) \times G^{g}(Z, Y) \cong G^{f \times g}(Z, X \times Y)=[Z, X \times Y]=$ $[Z, X] \times[Z, Y]$ and hence $G^{f}(Z, X)=[Z, X]$ and $G^{g}(Z, Y)=[Z, Y]$. Thus $X$ is a $W C_{k}^{f}$-space with respect to $Z$ and $Y$ is a $W C_{k}^{g}$-space with respect to $Z$.

Conversely, suppose that $X$ is a $W C_{k}^{f}$-space with respect to $Z$ and $Y$ is a $W C_{k}^{g}$-space with respect to $Z$. Then $G^{f}(Z, X)=[Z, X]$ and $G^{g}(Z, Y)=[Z, Y]$ by Theorem 2.11. It follows that $G^{f \times g}(Z, X \times Y) \cong$ $G^{f}(Z, X) \times G^{g}(Z, Y)=[Z, X] \times[Z, Y]=[Z, X \times Y]$. Thus $X \times Y$ is a $W C_{k}^{f \times g}$-space with respect to $Z$.

\section{3. $D W C_{k}^{p}$-spaces with respect to spaces}

In [3], Ganea introduced the concept of cocategory of a space as follows; Let $X$ be a any space. Define a sequence of cofibrations

$$
\mathcal{C}_{k}: X \stackrel{e_{k}^{\prime}}{\longrightarrow} F_{k} \stackrel{s_{k}^{\prime}}{\longrightarrow} B_{k}(k \geq 0)
$$

as follows, let $\mathcal{C}_{0}: X \stackrel{e_{0}^{\prime}}{\rightarrow} c X \stackrel{s_{0}^{\prime}}{\rightarrow} \Sigma X$ be the standard cofibration. Assuming $\mathcal{C}_{k}$ to be defined, let $F_{k+1}^{\prime}$ be the fibre of $s_{k}^{\prime}$ and $e_{k+1}^{\prime \prime}: X \rightarrow F_{k+1}^{\prime}$ lift $e_{k}^{\prime}$. Define $F_{k+1}$ as the reduced mapping cylinder of $e_{k+1}^{\prime \prime}$, let $e_{k+1}^{\prime}$ : $X \rightarrow F_{k+1}$ is the obvious inclusion map, and let $B_{k+1}=F_{k+1} / e_{k+1}^{\prime}(X)$ and $s_{k+1}^{\prime}: F_{k+1} \rightarrow F_{k+1} / e_{k+1}(X)$ the quotient map.

Definition 3.1. [3] The cocategory of $X$, cocat $X$, is the least integer $k \geq 0$ for which there is a map $r: F_{k} \rightarrow X$ such that $r \circ e_{k}^{\prime} \sim 1$. If there is no such integer, cocat $X=\infty$.

The following remark can easily obtained from the above definition. 
REMARK 3.2. cocat $X \leq k$ if and only if $e_{k}^{\prime}: X \rightarrow F_{k}$ has a left homotopy inverse.

For a map $p: X \rightarrow A$, a based map $g: X \rightarrow B$ is $p$-cocyclic [9] if there is a map $\theta: X \rightarrow A \vee B$ such that $j \theta \sim(p \times g) \Delta$, where $j: A \vee B \rightarrow A \times B$ is the inclusion and $\Delta: X \rightarrow X \times X$ is the diagonal map. The dual Gottlieb set for a map $p: X \rightarrow A, D G^{p}(X, B)$, is the set of all homotopy classes of $p$-cocyclic maps from $X$ to $B$. In the case $p=1_{X}: X \rightarrow X$, we call a 1-cocyclic map is just a cocyclic map, and denoted by, $D G(X, B)$, which is the set of all homotopy classes of cocyclic maps from $X$ to $B$.

In general, $D G(X, B) \subset D G^{p}(X, B) \subset[X, B]$ for any map $p: X \rightarrow$ $A$ and any space $B$. However, there is an example in [13] such that $D G(X, B) \neq D G^{p}(X, B) \neq[X, B]$.

Let $g: X \rightarrow Z$ be a map. A cocategory of a map is less than equal to $k$, cocat $g \leq k$,[3] if there is a map $\bar{g}: F_{k}^{X} \rightarrow Z$ such that $\bar{g} \circ e_{k}^{\prime X} \sim g: X \rightarrow Z$.

Definition 3.3. Let $X, Z$ be any two spaces. The mapcocategory of mapping space from $X$ to $Z$ is less than equal to $k$, mapcocat $(X, Z) \leq k$, means that for any map $g: X \rightarrow Z$, cocat $g \leq k$.

It is clear that mapcocat $(X, Z) \leq k$ if and only if $\left(e_{k}^{\prime X}\right)^{\#}:\left[F_{k}^{X}, Z\right] \rightarrow$ $[X, Z]$ is an epimorphism.

Proposition 3.4. cocat $Z \leq k$ if and only if for any space $X$, mapcocat $(X, Z) \leq k$.

Proof. Suppose that cocat $Z \leq k$. Then there is a map $s_{k}^{\prime}: F_{k}^{Z} \rightarrow Z$ ) such that $s_{k}^{\prime Z} \circ e_{k}^{\prime Z} \sim 1$. Let $X$ be a space and $g: X \rightarrow Z$ a map. We see $F_{k}(g) \circ e_{k}^{\prime X} \sim e_{k}^{\prime Z} \circ g$ by the naturality of the construction of $F_{k}^{Z}$ as is shown in the following homotopy commutative diagram:

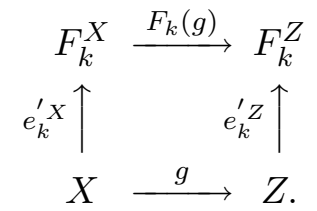

Thus we have a map $\bar{g}=s_{k}^{\prime} \cap \circ F_{k}(g): F_{k}^{X} \rightarrow Z$ such that $\bar{g} \circ e_{k}^{\prime X} \sim g$. Thus we know mapcocat $(X, Z) \leq k$. On the other hand, suppose that for any space $X$, the mapcocategory mapcocat $(X, Z) \leq k$. Taking $X=Z$ and $g=1_{Z}$, then we know that cocat $Z \leq k$.

Proposition 3.5. cocat $X \leq k$ if and only if for any space $Z$, mapcocat $(X, Z) \leq k$. 
Proof. Suppose that cocat $X \leq k$. Then there is a map $s_{k}^{\prime X}: F_{k}^{X} \rightarrow X$ such that $s_{k}^{\prime X} \circ e_{k}^{\prime X} \sim 1$. Let $Z$ be a space and $g: X \rightarrow Z$ a map. Then we have $\left(g \circ s_{k}^{\prime X}\right) \circ e_{k}^{\prime X} \sim 1_{X} \circ g \sim g$ and mapcat $(X, Z) \leq k$. On the other hand, suppose that for any space $Z$, the mapcocategory mapcocat $(X, Z) \leq k$. Taking $Z=X$ and $g=1_{X}$, then we know that cocat $X \leq k$.

It is introduced [18] that a space $X$ is called $D C_{k}^{p}$-space for a map $p: X \rightarrow A$ if $e_{k}^{\prime X}: X \rightarrow F_{k}^{X}$ is $p$-cocyclic.

Lemma 3.6. Let $p: X \rightarrow A$ be a map. Then $g: X \rightarrow B$ is p-cocyclic if and only if $(g)^{\#}([B, Z]) \subset D G^{p}(X, Z)$ for any space $Z$.

Proof. Suppose that $g: X \rightarrow B$ is $p$-cocyclic. Let $Z$ be a space and $h: B \rightarrow Z$ a map. Since $g: X \rightarrow B$ is $p$-cocyclic, there is a map $\theta: X \rightarrow$ $A \vee B$ such that $j \theta \sim(p \times g) \Delta$, where $j: A \vee B \rightarrow A \times B$ is the inclusion and $\Delta: X \rightarrow X \times X$ is the diagonal map. Then $\phi=(1 \vee h) \theta: X \rightarrow A \vee Z$ satisfies $j \phi \sim(p \times h g) \Delta$. Thus we have $g^{\#}([B, Z]) \subset D G^{p}(X, Z)$ for any space $Z$. On the other hand, taking $Z=B$ and $1_{B}: B \rightarrow B \in[B, B]$. Since $g \sim g^{\#}\left(1_{B}\right) \in D G^{p}(X, B), g: X \rightarrow B$ is $p$-cocyclic.

Theorem 3.7. [18] $A$ space $X$ is a $D C_{k}^{p}$-space for a map $p: X \rightarrow A$ if and only if $D G^{p}(X, Z)=[X, Z]$ for any space $Z$ with cocat $Z \leq k$.

REMARK 3.8. If a space $Z$ satisfy cocat $Z \leq k$, then also $Z$ is a space with mapcocat $(Z, X) \leq k$ from Proposition 3.4.

Definition 3.9. Let $p: X \rightarrow A$ be a map and $Z$ a space with mapcocat $(X, Z) \leq k$. A space $X$ is called a $D W C_{k}^{p}$-space with respect to a space $Z$ if $\left(e_{k}^{\prime X}\right)^{\#}\left(\left[F_{k}^{X}, Z\right]\right) \subset D G^{p}(X, Z)$.

Theorem 3.10. Let $p: X \rightarrow A$ be a map and $Z$ a space with mapcocat $(X, Z) \leq k$. Then a space $X$ is a $D W C_{k}^{p}$-space with respect to $Z$ if and only if $D G^{p}(X, Z)=[X, Z]$.

Proof. Suppose that $X$ is a $D W C_{k}^{p}$-space with respect to $Z$. Since mapcocat $(X, Z) \leq k,\left(e_{k}^{\prime}\right)^{\#}:\left[F_{k}^{X}, Z\right] \rightarrow[X, Z]$ is an epimorphism. Since $X$ is a $D W C_{k}^{p}$-space with respect to $Z,[X, Z]=\left(e_{k}^{\prime X}\right)^{\#}\left(\left[F_{k}^{X}, Z\right]\right) \subset$ $D G^{p}(X, Z)$ and $D G^{p}(X, Z)=[X, Z]$.

Conversely, assume that $D G^{p}(X, Z)=[X, Z]$. Thus we know $\left(e_{k}^{\prime X}\right)^{\#}$ $\left(\left[F_{k}^{X}, Z\right]\right)=[X, Z] \subset G^{p}(X, Z)$ and $X$ is a $D W C_{k}^{p}$-space with respect to $Z$.

We have the following corollary from Theorem 3.7 and Remark 3.8. 
Corollary 3.11. $X$ is a $D C_{k}^{p}$-space if and only if for each space $Z$ with cocat $Z \leq k, X$ is a $D W C_{k}^{p}$-space with respect to $Z$.

Let $p: X \rightarrow A$ and $q: Y \rightarrow A$ be any maps. Then it is known [18] that the relation $D G^{\nabla(p \vee q)}(X \vee Y, B) \equiv D G^{p}(X, B) \times D G^{q}(Y, B)$ holds for any space $B$.

Theorem 3.12. Let $p: X \rightarrow A$ and $q: Y \rightarrow A$ be any maps and $Z$ a space with mapcocat $(X, Z) \leq k$. Then the wedge space $X \vee Y$ is a $D W C_{k}^{\nabla(p \vee q)}$-space with respect to $Z$ if and only if $X$ is a $D W C_{k}^{p}$-space with respect to $Z$ and $Y$ is a $D W C_{k}^{q}$-space with respect to $Z$.

Proof. If $X \vee Y$ is a $D W C_{k}^{\nabla(p \vee q)}$-space with respect to $Z$, then we know, from Theorem 3.10 and the above fact, that $D G^{p}(X, Z) \times D G^{q}(Y$, $Z) \equiv D G^{\nabla(p \vee q)}(X \vee Y, Z)=[X \vee Y, Z] \equiv[X, Z] \times[Y, Z]$. Then we have $D G^{p}(X, Z)=[X, Z]$ and $D G^{q}(Y, Z)=[Y, Z]$. Thus we know that $X$ is a $D W C_{k}^{p}$-space with respect to $Z$ and $Y$ is a $D W C_{k}^{q}$-space with respect to $Z$. On the other hand, suppose that $X$ is a $D W C_{k}^{p}$-space with respect to $Z$ and $Y$ is a $D W C_{k}^{q}$-space with respect to $Z$. Then $D G^{p}(X, Z)=$ $[X, Z], D G^{q}(Y, Z)=[Y, Z]$. Thus we know $D G^{\nabla(p \vee q)}(X \vee Y, Z) \equiv$ $D G^{p}(X, Z) \times D G^{q}(Y, Z)=[X, Z] \times[Y, Z] \equiv[X \vee Y, Z]$. Thus $X \vee Y$ is a $D W C_{k}^{\nabla(p \vee q)}$-space with respect to $Z$.

\section{References}

[1] J. Aguadé, Decomposable free loop spaces, Can. J. Math. 39 (1987), 938-955.

[2] T. Ganea, Lusternik-Schnirelmann category and cocategory, Proc. London Math. Soc. (3)10 (1960), 623-639.

[3] T. Ganea, A generalization of the homology and homotopy suspension, Comment. Math. Helv., 39(1965), 295-322.

[4] N. Iwase, Ganea's conjecture on Lusternik-Schnirelmann category, Bull. Lon. Math. Soc. 30 (1998), 623-634.

[5] N. Iwase, M. Mimura, N. Oda and Y. S. Yoon, The Milnor-Stasheff filtration on spaces and generalized cyclic maps, Canad. Math. Bull. 55 (2012), no. 3, $523-536$.

[6] I. M. James, On category in the sense of Lusternik-Schnirelmann, Topology 17 (1978), 331-348.

[7] K. L. Lim, Cocyclic maps and coevaluation subgroups, Canad. Math. Bull. 30 (1987), 63-71.

[8] J. Milnor, Construction of universal bundles, I, II, Ann. Math. 63 (1956), $272-284,430-436$.

[9] N. Oda, The homotopy of the axes of pairings, Canad. J. Math. 17 (1990), $856-868$. 
[10] J. D. Stasheff, Homotopy associativity of H-spaces I, II, Trans. Amer. Math. Soc. 108 (1963), 275-292, 293-312.

[11] K. Varadarajan, Genralized Gottlieb groups, J. Indian Math. Soc. 33 (1969), 141-164.

[12] M. H. Woo and Y. S. Yoon, T-spaces by the Gottlieb groups and duality, J. Austral. Math. Soc. (Series A) 59 (1995), 193-203.

[13] Y. S. Yoon, The generalized dual Gottlieb sets, Top. Appl. 109 (2001), 173-181.

[14] Y. S. Yoon, Generalized Gottlieb groups and generalized Wang homomorphisms, Sci. Math. Japon. 55 (2002), no. 1, 139-148.

[15] Y. S. Yoon, $H^{f}$-spaces for maps and their duals, J. Korea Soc. Math. Educ. Ser. B 14 (2007), no. 4, 289-306.

[16] Y. S. Yoon, Lifting T-structures and their duals, J. Chungcheong Math. Soc. 20 (2007), no. 3, 245-259.

[17] Y. S. Yoon, On cocyclic maps and cocategory, J. Chungcheong Math. Soc. 24 (2011), no. 1, 137-140.

[18] Y. S. Yoon and H. D. Kim, Generalized dual Gottlieb sets and cocategories, J. Chungcheong Math. Soc. 25 (2012), no. 1, 135-140.

Department of Mathematics Education

Hannam University

Daejeon 306-791, Republic of Korea

E-mail: yoon@hannam.ac.kr 